\title{
ON THE GROWTH OF MEROMORPHIC FUNCTIONS
}

\author{
By Mitsuru Ozawa
}

$\S 1$. Introduction. By making use of Fourier series method Miles and Shea [4], [5] recently obtained a better estimate for the $\kappa(\lambda)$ and related results. It seems to the present author that the method contains more. In this paper we shall discuss some of them.

For completeness we shall list up several known results, which will be used later. For a meromorphic function $f(z)$ we define $m_{2}(r, f)$ by

$$
m_{2}(r, f)^{2}=\frac{1}{2 \pi} \int_{0}^{2 \pi}\left(\log \left|f\left(r e^{i \theta}\right)\right|\right)^{2} d \theta .
$$

In what follows we only consider entire or meromorphic functions of the following form:

$$
f(z)=\Pi E\left(\frac{z}{z_{\nu}}, q\right)
$$

or

where

$$
f(z)=\Pi E\left(\frac{z}{z_{\nu}}, q\right) / \Pi E\left(\frac{z}{w_{\nu}}, q\right)
$$

$$
E(x, q)=(1-x) \exp \left(x+x^{2} / 2+\cdots+x^{q} / q\right), \quad q=[\lambda]
$$

and $\lambda$ is the order of $f(z), \lambda<\infty$.

Let $c_{m}(r)$ be the $m$-th Fourier coefficient:

$$
c_{m}(r)=\frac{1}{2 \pi} \int_{0}^{2 \pi}\left(\log \left|f\left(r e^{i \theta}\right)\right|\right) e^{-\imath m \theta} d \theta .
$$

Then

$$
m_{2}(r, f)^{2}=\sum_{m=-\infty}^{\infty}\left|c_{m}(r)\right|^{2} .
$$

Edrei and Fuchs [1] had shown that, with $r_{\nu}=\left|z_{\nu}\right|$ and $s_{\nu}=\left|w_{\nu}\right|$,

$$
c_{m}(r)=-\frac{1}{2 m} \sum_{r_{\nu} \leqq r}\left\{\left(\frac{r}{z_{\nu}}\right)^{m}-\left(\frac{\bar{z}_{\nu}}{r}\right)^{m}\right\}-\frac{1}{2 m} \sum_{s_{\nu} \leqq r}\left\{\left(\frac{r}{w_{\nu}}\right)^{m}-\left(\frac{\bar{w}_{\nu}}{r}\right)^{m}\right\}
$$

for $m \geqq 1$ and, for $m \geqq q+1$,

$$
c_{m}(r)=-\frac{1}{2 m}\left\{\sum_{r_{\nu}>r}\left(\frac{r}{z_{\nu}}\right)^{m}-\sum_{s_{\nu}>r}\left(\frac{r}{w_{\nu}}\right)^{m}+\sum_{r_{\nu} \leqq r}\left(\frac{\bar{z}_{\nu}}{r}\right)^{m}-\sum_{s_{\nu} \leqq r}\left(\frac{\bar{w}_{\nu}}{r}\right)^{m}\right\} .
$$

Received January 7, 1983 
Obviously $c_{m}(r)=\bar{c}_{-m}(r)$ for $m \leqq-1$ and $c_{0}(r)=N(r, 0, f)-N(r, \infty, f)$. Further For $m \geqq q+1$

$$
\left|c_{m}(r)\right| \leqq 2 T(r, f)-N(r, 0, f)-N(r, \infty, f) \leqq m_{2}(r, f) .
$$

$$
\begin{aligned}
& -\frac{1}{2 m}\left\{\sum_{r_{\nu}>r}\left(\frac{r}{r_{\nu}}\right)^{m}+\sum_{r_{\nu} \leqq r}\left(\frac{r_{\nu}}{r}\right)^{m}\right\} \\
& =N(r, 0)-\frac{m}{2} \int_{0}^{r} \frac{N(t, 0)}{t}\left(\frac{t}{r}\right)^{m} d t-\frac{m}{2} \int_{r}^{\infty} \frac{N(t, 0)}{t}\left(\frac{r}{t}\right)^{m} d t
\end{aligned}
$$

and for $m \leqq q$

$$
\begin{aligned}
& \frac{1}{2 m} \sum_{r_{\nu} \leqq r}\left\{\left(\frac{r}{r_{\nu}}\right)^{m}-\left(\frac{r_{\nu}}{r}\right)^{m}\right\} \\
& \quad=N(r, 0)+\frac{m}{2} \int_{0}^{r} \frac{N(t, 0)}{t}\left\{\left(\frac{r}{t}\right)^{m}-\left(\frac{t}{r}\right)^{m}\right\} d t .
\end{aligned}
$$

$\S 2$. Discussion of results. Our first result is the following

THEOREM 1. Let $f(z)$ be the canonical product formed by $\left\{z_{2}\right\}$, which satısfies

$$
\sum\left|z_{\nu}\right|^{-q}=\infty, \quad \sum\left|z_{\nu}\right|^{-q-1}<\infty
$$

for a positive integer $q$ and

Then

$$
\left|\arg z_{\nu}\right| \leqq \omega, \quad 0 \leqq \omega \leqq(\pi-\varepsilon) / 2 q, \quad 0<\varepsilon \leqq \pi .
$$

$$
\varlimsup_{r \rightarrow \infty} \frac{N(r, 0, f)}{m_{2}(r, f)} \leqq \frac{1}{A(q, \omega)}
$$

and

$$
\varlimsup_{r \rightarrow \infty} \frac{m(r, f)}{m_{2}(r, f)} \leqq \frac{1}{2}+\frac{1}{2 A(q, \omega)}
$$

where

$$
A(q, \omega)=\left\{q+1+\frac{\cos (q+1) \omega \sin q \omega}{\sin \omega}\right\}^{1 / 2},
$$

Since $m_{2}(r, f) \geqq N(r, 0, f)$ for entire functions with $f(0)=1$,

$$
\varlimsup_{r \rightarrow \infty} \frac{N(r, 0, f)}{m_{2}(r, f)} \leqq B
$$

loses its effectivity when $B \geqq 1$. We shall prove that the estimate given in Theorem 1 loses its efficiency when $q=1$ and $\omega=\pi / 2$. Further for entire $f$

$$
\varlimsup_{r \rightarrow \infty} \frac{N(r, 0, f)}{m_{2}(r, f)} \leqq \varlimsup_{r \rightarrow \infty} \frac{N(r, 0, f)}{2 m(r, f)-N(r, 0, f)-O(1)}
$$




$$
=\frac{1-\delta(0, f)}{1+\delta(0, f)} \text {. }
$$

This estimate is effective when $\delta(0, f)>0$. In this direction Kobayashi [2] had shown the following

THEOREM A. Under the same assumptions as in Theorem 1 with $\omega=\pi / 2(q+1)$

$$
\delta(0, f)>0 \text {. }
$$

If $\omega>\pi / 2(q+1)$, then there is an entıre function such that $\delta(0, f)=0$.

If $0<\varepsilon<\pi /(q+1)$, then $(\pi-\varepsilon) / 2 q>\pi / 2(q+1)$. Therefore $\delta(0, f)=0$ does not always imply the inefficiency of our Theorem 1. The opening of $\omega$ in Theorem 1 is equal to the one of the following result due to Kobayashi [3].

THEOREM B. Under the same assumptions as in Theorem 1

$$
q \leqq \mu \leqq \lambda \leqq q+1,
$$

where $\lambda$ and $\mu$ are the order and the lower order of $f$, respectively. This is best possible.

Theorem 1 can be extended to a wider opening if $q \geqq 2$. For example, if $q=2$ and $\omega=(\pi-\varepsilon) / 2(\varepsilon>0)$, then

$$
\begin{aligned}
m_{2}(r, f)^{2} & \geqq\left|c_{0}(r)\right|^{2}+2\left|c_{1}(r)\right|^{2} \\
& \geqq N(r, 0, f)^{2}\left(1+2 \sin ^{2} \varepsilon\right) .
\end{aligned}
$$

This gives an estimate of desired type.

THEOREM 2. Let $f(z)$ be the canonical product formed by the set of zeros $\left\{a_{\nu},-a_{\nu}\right\}$. Assume that

Then

$$
\begin{gathered}
\Sigma\left|a_{\nu}\right|^{-q}=\infty, \quad \sum\left|a_{\nu}\right|^{-q-1}<\infty, \\
\left|\arg a_{\nu}\right| \leqq \omega, \quad 0 \leqq \omega \leqq(\pi-\varepsilon) / 2 q, \quad \varepsilon>0 .
\end{gathered}
$$

$$
\varlimsup_{r \rightarrow \infty} \frac{N(r, 0, f)}{m_{2}(r, f)} \leqq \frac{1}{A}
$$

where

$$
A^{2}=1+\left[\frac{q}{2}\right]+\frac{\cos ([q / 2]+1) \omega \sin [q / 2] \omega}{\sin 2 \omega} .
$$

This theorem has its meaning only if $q \geqq 2$.

THEOREM 3. Let $f(z)$ be a meromorphic function being representable as 
$f_{1}(z) / f_{2}(z)$, where $f_{1}$ and $f_{2}$ are canonical products formed by $\left\{a_{n}\right\}$ and $\left\{b_{n}\right\}$, respectively. Assume that

and

$$
\sum\left|a_{n}\right|^{-q}=\infty, \quad \Sigma\left|b_{n}\right|^{-q}=\infty, \quad \sum\left|a_{n}\right|^{-q-1}+\Sigma\left|b_{n}\right|^{-q-1}<\infty
$$

$$
\left|\arg a_{n}\right| \leqq \omega, \quad\left|\pi-\arg b_{n}\right| \leqq \omega
$$

with $0 \leqq \omega \leqq(\pi-\varepsilon) / 2 q$. Then

$$
\varlimsup_{r \rightarrow \infty} \frac{N(r, 0, f)+N(r, \infty, f)}{m_{2}(r, f)} \leqq \frac{1}{A},
$$

where

$$
A^{2}=s+1+\frac{\sin 4(s+1) \omega}{2 \sin 2 \omega}, \quad s=\max \{p \mid 2 p+1 \leqq q\}
$$

In [5] Miles and Shea indicated that $m_{2}(r, f) \leqq 4 \sqrt{ } q+1 m(r, f)$, if $f$ is entire, of finite genus $q$ with only positive zeros. More precisely,

where

$$
\begin{aligned}
m_{2}(r, f)^{2} & \leqq 8\left(q+s_{q}\right) m(r, f)^{2}-8\left(q+s_{q}\right) m(r, f) N(r, 0, f)+(2 q+2 s+1) N(r, 0, f)^{2} \\
& \leqq 8\left(q+s_{q}\right) m(r, f)^{2},
\end{aligned}
$$

$$
s_{q}=(q+1)^{2} \sum_{j=0}^{\infty} \frac{1}{(q+1+j)^{2}}=(q+1)^{2}\left(\frac{\pi^{2}}{6}-\sum_{j=1}^{q} \frac{1}{j^{2}}\right) .
$$

It is very easy to prove $q+1<s_{q}<q+2$.

Let $f$ be the canonical product of genus $q$. When does the estimate $m_{2}(r, f) \leqq K m(r, f), \quad 0<K<\infty$, hold ? Of course this does not hold in general. Let us denote

$$
f(z)=\Pi E\left(\frac{z}{a_{\nu}}, q\right), \quad F(z)=\Pi E\left(\frac{z}{\left|a_{\nu}\right|}, q\right) .
$$

If the Valiron deficiency $\Delta(0, F)$ of $F$ satisfies $\Delta(0, F)<1$, then $m_{2}(r, f) \leqq K m(r, f)$ and $(1-\Delta(0, F)-\varepsilon) m(r, F)<N(r, 0, F)=N(r, 0, f) \leqq m(r, f)$ give the result. Since $m_{2}(r, F) \leqq k m(r, F)$ holds without any condition, $\Delta(0, F)<1$ is not a necessary condition. So it is hoped to give a more appropriate condition for the above problem.

In the above results we do not make use of the concept of Pólya peaks of any kind. Under the assumptions of Theorem 1 we can prove

$$
\varlimsup_{r \rightarrow \infty} \frac{N(r, 0, f)}{m_{2}(r, f)} \leqq \frac{1}{A^{1 / 2}}, \quad A=1+2 \sum_{1}^{q}\left(\frac{\rho^{2}}{\rho^{2}-m^{2}}\right)^{2} \cos ^{2} m \omega
$$

by making use of Pólya peaks of the second kind, order $\rho$, for $N(r, 0, f)$. We can also prove similar results corresponding to Theorem 2 and Theorem 3 quite similarly. 
Let $M_{\rho}$ be the class of meromorphic functions $f(z)$ of order $\rho$ defined by $f_{1}(z) / f_{1}(-z)$ with the canonical product

$$
f_{1}(z)=\Pi E\left(\frac{z}{a_{n}}, q\right), \quad q=[\rho] .
$$

Let $F(z)$ be $F_{1}(z) / F_{1}(-z)$ with

$$
F_{1}(z)=\Pi E\left(\frac{z}{\left|a_{n}\right|}, q\right) .
$$

THEOREM 4. Let $f(z)$ belong to $M_{\rho}$. Then $m_{2}(r, f) \leqq m_{2}(r, F)$ ana

$$
\varlimsup_{r \rightarrow \infty} \frac{N(r, 0, f)}{m_{2}(r, f)} \geqq \frac{\sqrt{2}}{\sqrt{ } \pi \rho} \frac{|\cos \pi \rho / 2|}{(\pi \rho-\sin \pi \rho)^{1 / 2}} .
$$

This is best possible.

THEOREM 5. Under the same assumptions as in Theorem 3 with $\omega=0$

$$
\varlimsup_{r \rightarrow \infty} \frac{N(r, 0, f)+N(r, \infty, f)}{m_{2}(r, f)} \leqq \frac{2 \sqrt{2}}{\sqrt{\pi \rho}} \frac{|\cos \pi \rho / 2|}{(\pi \rho-\sin \pi \rho)^{1 / 2}} .
$$

Thus is best possible.

§3. Proof of Theorem 1. For $1 \leqq m \leqq q$

Hence

$$
\begin{gathered}
\mathscr{R} c_{m}(r)=\frac{1}{2 m} \sum_{r_{\nu} \leqq r}\left\{\left(\frac{r}{r_{\nu}}\right)^{m}-\left(\frac{r_{\nu}}{r}\right)^{m}\right\} \cos m \varphi_{\nu}, \\
z_{\nu}=r_{\nu} e^{2 \varphi_{\nu}}, \quad\left|\varphi_{\nu}\right| \leqq \omega .
\end{gathered}
$$

Thus

$$
\begin{aligned}
\mathscr{R} c_{m}(r) & \geqq \frac{1}{2 m} \sum_{r_{\nu} \unlhd r}\left\{\left(\frac{r}{r_{\nu}}\right)-\left(\frac{r_{\nu}}{r}\right)^{m}\right\} \cdot \cos m \omega \\
& =\cos m \omega\left[N(r, 0, f)+\frac{m}{2} \int_{0}^{r}\left\{\left(\frac{r}{t}\right)^{m}-\left(\frac{t}{r}\right)^{m}\right\} \frac{N(t, 0, f)}{t} d t\right. \\
& \geqq N(r, 0, f) \cos m \omega .
\end{aligned}
$$

$$
\begin{aligned}
m_{2}(r, f)^{2} & \geqq N(r, 0, f)^{2}\left(1+2 \sum_{m=1}^{q} \cos ^{2} m \omega\right) \\
& =N(r, 0, f)^{2}\left(q+1+\frac{\cos (q+1) \omega \sin q \omega}{\sin \omega}\right) .
\end{aligned}
$$

This gives the first desired result. By $m_{2}(r, f) \geqq 2 m(r, f)-N(r, 0, f)$ we have the second desired result.

If $\omega=0$, the $A(q, 0)^{2}=2 q+1$ and

$$
\varlimsup_{r \rightarrow \infty} \frac{N(r, 0, f)}{m_{2}(r, f)} \leqq \frac{1}{\sqrt{2 q+1}} .
$$


$\S 4$. Proof of Theorem 2. In this case for $2 s+1 \leqq q c_{2 s+1}(r)=0$. Further

$$
\begin{aligned}
\mathscr{R} c_{2 s}(r) & =\frac{1}{2 s} \mathscr{R} \sum_{\left|a_{\nu}\right| \leqq r}\left\{\left(\frac{r}{a_{\nu}}\right)^{2 s}-\left(\frac{a_{\nu}}{r}\right)^{2 s}\right\} \\
& \geqq \frac{\cos 2 s \omega}{2 s} \sum_{\left|a_{\nu}\right| \leqq r}\left\{\left(\frac{r}{\left|a_{\nu}\right|}\right)^{2 s}-\left(\frac{\left|a_{\nu}\right|}{r}\right)^{2 s}\right\},
\end{aligned}
$$

where $\sum_{\left|a_{\nu}\right| \leqq r}$ means the summation over all $a_{\nu}$ but not over any $-a_{\nu}$. Hence

Therefore

$$
\begin{aligned}
\mathscr{R} c_{2 s}(r) & \geqq \cos 2 s \omega\left[N(r, 0, f)+s \int_{0}^{r}\left\{\left(\frac{r}{t}\right)^{2 s}-\left(\frac{t}{r}\right)^{2 s}\right\} \frac{N(t, 0, f)}{t} d t\right. \\
& \geqq N(r, 0, f) \cos 2 s \omega .
\end{aligned}
$$

$$
\begin{aligned}
m_{2}(r, f)^{2} & \geqq\left\{1+2 \sum_{1}^{[q / 2]} \cos ^{2} 2 s \omega\right\} N(r, 0, f)^{2} \\
& =\left\{1+\left[\frac{q}{2}\right]+\frac{\cos ([q / 2]+1) \omega \sin [q / 2] \omega}{\sin 2 \omega}\right\} N(r, 0, f)^{2} .
\end{aligned}
$$

This gives the desired result.

If $\omega=0$ holds, then we can prove that

$$
\begin{aligned}
m_{2}(r, f)^{2} \leqq & 4\left(2 s_{0}-2+2 s_{q}\right)\left(m(r, f)^{2}-m(r, f) N(r, 0, f)\right) \\
& +\left(2 s_{0}-1+2 s_{q}\right) N(r, 0, f)^{2},
\end{aligned}
$$

where $s_{0}=\min \{s \mid 2 s \geqq q+1\}$ and

$$
s_{q}=s_{0}^{2} \sum_{j=0}^{\infty}\left(s_{0}+j\right)^{-2} .
$$

§5. An example. Let $a$, be

$$
2^{j / \rho}
$$

Let $f(z)$ be the canonical product formed by $\left\{a_{\jmath},-a_{\jmath}\right\}_{\jmath=1,2, \ldots}$ with their multiplicities $a_{j}^{\rho}$. Evidently for $\varepsilon>0$

$$
\sum \frac{a_{j}^{\rho}}{a_{\jmath}^{\rho}}=\infty, \quad \sum \frac{a_{j}^{\rho}}{a_{j}^{\rho+\varepsilon}} \leqq \sum a_{\jmath}^{-\hat{\varepsilon}}<\infty
$$

Hence the exponent of convergence of the given series is equal to $\rho$. Assume that $1<\rho<2$. Then $f(z)$ is of the first genus. In the present case

$$
f(z)=\prod_{\jmath=1}^{\infty}\left(1-\frac{z^{2}}{a_{\jmath}^{2}}\right)^{a_{\jmath}^{\rho}} .
$$

Let $n(r)$ be the number of zeros of $f(z)$ in $|z| \leqq r$ and $N(r)$ the counting function of zeros of $f(z)$ there. Then for $a_{p} \leqq r<a_{p+1}$ 


$$
n(r)=2 \sum_{j=1}^{p} a_{\jmath}^{\rho}
$$

and

$$
\begin{aligned}
N(r) & =\int_{0}^{r} \frac{n(t)}{t} d t \\
& =2 \sum_{k=1}^{p-1} \log \frac{a_{k+1}}{a_{k}} \sum_{j=1}^{k-1} a_{j}^{\rho}+2 \log \frac{r}{a_{p}} \sum_{j=1}^{p} a_{\jmath}^{\rho} .
\end{aligned}
$$

Now we put $r_{p}=\left(a_{p} a_{p+1}^{2-\rho}\right)^{1 / 2}$. Then

$$
N\left(r_{p}\right) \sim a_{p}^{\rho} \log \left(a_{p}^{-1} a_{p+1}^{2-\rho}\right),
$$

which is very easy to prove. Let $M(r, f)$ and $m^{*}(r, f)$ be the maximum modulus and the minimum modulus of $f(z)$ on $|z|=r$. Then

$$
\begin{aligned}
& \log M(r, f)=\log |f(i r)|, \\
& \log m^{*}(r, f)=\log |f(r)| .
\end{aligned}
$$

Then we can prove that

$$
\log M\left(r_{p}, f\right) \sim a_{p}^{\rho} \log \left(a_{p}^{-1} a_{p+1}^{2-\rho}\right)
$$

and

$$
\log m^{*}\left(r_{p}, f\right) \sim a_{p}^{\rho} \log \left(a_{p}^{-1} a_{p+1}^{2-\rho}\right) .
$$

Therefore along the sequence $\left\{r_{p}\right\}$

Hence

$$
m_{2}(r, f) \sim \log M(r, f) \sim \log m^{*}(r, f) \sim N(r, 0, f) .
$$

$$
\varlimsup_{r \rightarrow \infty} \frac{N(r, 0, f)}{m_{2}(r, f)} \geqq \varlimsup_{p \rightarrow \infty} \frac{N\left(r_{p}, 0, f\right)}{m_{2}\left(r_{p}, f\right)}=1 .
$$

Thus we have the desired non-effectivity of estimations in Theorem 1 for $q=1$, $\omega=\pi / 2$ and in Theorem 2 for $q=1, \omega=0$.

§6. Proof of Theorem 3. In this case for $2 p+1 \leqq q$

$$
\begin{aligned}
\mathscr{R} c_{2 p+1}(r) \geqq & \frac{1}{2(2 p+1)}\left[\sum_{r_{\nu} \leqq r}\left\{\left(\frac{r}{r_{\nu}}\right)^{2 p+1}-\left(\frac{r_{\nu}}{r}\right)^{2 p+1}\right\} \cos (2 p+1) \theta_{\nu}\right. \\
& -\sum_{t_{\nu} \leqq r}\left\{\left(\frac{r}{t_{\nu}}\right)^{2 p+1}-\left(\frac{t_{\nu}}{r}\right)^{2 p+1}\right\} \cos (2 p+1) \varphi_{\nu},
\end{aligned}
$$

where $\left|\theta_{\nu}\right| \leqq \omega,\left|\pi-\varphi_{\nu}\right| \leqq \omega$. Hence

$$
\mathscr{R} c_{2 p+1}(r) \geqq(N(r, 0)+N(r, \infty)) \cos (2 p+1) \omega .
$$

Further with $s=\max \{p \mid 2 p+1 \leqq q\}$ 


$$
\begin{aligned}
m_{\mathbf{2}}(r, f)^{2} & \geqq 2 \sum_{0}^{s}\left|c_{2 p+1}(r)\right|^{2} \\
& \geqq 2(N(r, 0)+N(r, \infty))^{2} \sum_{0}^{s} \cos ^{2}(2 p+1) \omega \\
& =(N(r, 0)+N(r, \infty))^{2}\left(s+1+\frac{\sin 4(s+1) \omega}{2 \sin 2 \omega}\right) .
\end{aligned}
$$

This is the desired result.

$\S 7$. Proof of Theorem 4. In this case $c_{2 p}(r)=0$ and for $2 p+1 \leqq q$

$$
\left|c_{2 p+1}(r)\right| \leqq \frac{1}{2 p+1}\left[\sum_{r_{\nu} \leqq r}\left\{\left(\frac{r}{r_{\nu}}\right)^{2 p+1}-\left(\frac{r_{\nu}}{r}\right)^{2 p+1}\right\}\right] \equiv \gamma_{2 p+1}(r)
$$

and for $2 p+1 \geqq q+1$

$$
\left|c_{2 p+1}(r)\right| \leqq \frac{1}{2 p+1}\left[\sum_{r_{\nu}>r}\left(\frac{r}{r_{\nu}}\right)^{2 p+1}+\sum_{r_{\nu} \leqq r}\left(\frac{r_{\nu}}{r}\right)^{2 p+1}\right\} \equiv \gamma_{2 p+1}(r) .
$$

Hence

$$
\begin{aligned}
m_{2}(r, f)^{2} & =2 \sum_{p=1}^{\infty}\left|c_{2 p-1}(r)\right|^{2} \\
& \leqq 2 \sum_{p=1}^{\infty} \gamma_{2 p-1}(r)^{2}=m_{2}(r, F)^{2} .
\end{aligned}
$$

Therefore it is sufficient to prove the result for $F$ instead of $f$. Then we can make use of the integral representation of $\gamma_{2 p-1}(r)$. Let $\left\{t_{n}\right\}$ be a sequence of Pólya peaks of the first kind, order $\rho$, for $N(r) \equiv N(r, 0)$. Then

Then we have

$$
\begin{array}{ll}
N(t) t_{n}^{\rho-\varepsilon} \leqq N\left(t_{n}\right) t^{\rho-\varepsilon}, & 0<t \leqq t_{n} \\
N(t) t_{n}^{\rho+\varepsilon} \leqq N\left(t_{n}\right) t^{\rho+\varepsilon}, & t_{n} \leqq t<\infty .
\end{array}
$$

$$
\gamma_{2 p-1}\left(t_{n}\right) \leqq 2 N\left(t_{n}\right) \frac{\rho^{2}+\varepsilon(2 p-1-\varepsilon)}{(2 p-1-\varepsilon)^{2}-\rho^{2}}, \quad 2 p-1 \geqq q+1
$$

and

$$
\gamma_{2 p-1}\left(t_{n}\right) \leqq 2 N\left(t_{n}\right) \frac{(\rho-\varepsilon)^{2}}{(\rho-\varepsilon)^{2}-(2 p-1)^{2}}, \quad 2 p-1 \leqq q .
$$

Hence

$$
\begin{aligned}
m_{2}\left(t_{n}, F\right)^{2} \leqq & 8 N\left(t_{n}\right)^{2}\left[\sum_{p=1}^{s}\left\{\frac{(\rho-\varepsilon)^{2}}{(\rho-\varepsilon)^{2}-(2 p-1)^{2}}\right\}^{2}\right. \\
& \left.+\sum_{p=s+1}^{\infty}\left\{\frac{\rho^{2}+\varepsilon(2 p-1-\varepsilon)}{(2 p-1-\varepsilon)^{2}-\rho^{2}}\right\}^{2}\right] .
\end{aligned}
$$

If $\varepsilon$ tends to zero, then the term in the bracket tends to 
which is equal to

$$
\sum_{p=1}^{\infty} \frac{(\rho / 2)^{4}}{\left\{(\rho / 2)^{2}-(p-1 / 2)^{2}\right\}^{2}},
$$

$$
\frac{1}{8}-\frac{\pi \rho-\sin \pi \rho}{\cos ^{2} \frac{\pi}{2} \rho} \cdot \frac{\pi}{2} \rho .
$$

This gives the desired result:

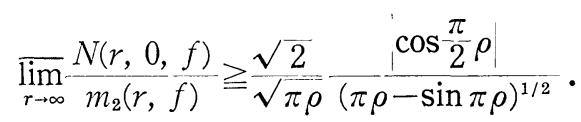

§ 8. Proof of Theorem 5. Firstly we have

$$
m_{2}(r, f)^{2} \geqq 2 \Sigma\left|c_{2 p+1}(r)\right|^{2} .
$$

Let us put $N(r)=N(r, 0, f)+N(r, \infty, f)$. We can make use of the integral representation of $c_{2 p+1}(r)$. Let $\left\{t_{n}\right\}$ be a sequence of Pólya peaks of the second kind, order $\rho$, for $N(r)$. Let $\left\{s_{n}\right\}$ and $\left\{S_{n}\right\}$ be the associated sequences such that $s_{n} \rightarrow \infty, t_{n} / s_{n} \rightarrow \infty, S_{n} / t_{n} \rightarrow \infty$ and

$$
N(t) \geqq(1+o(1))\left(t / t_{n}\right)^{\rho} N\left(t_{n}\right)
$$

for $s_{n} \leqq t \leqq S_{n}$. Similarly as in the proof of Theorem 4

$$
m_{2}\left(t_{n}, f\right)^{2} \geqq 2 N\left(t_{n}\right)^{2} \sum_{p=1}^{\infty}\left(\frac{\rho^{2}}{\rho^{2}-(2 p-1)^{2}}\right)^{2}(1+o(1)) .
$$

Hence we have the desired result.

$\S 9$. In this section we shall give an extension of Theorem 4 . Let $f(z)$ be the canonical product formed by zeros $\left\{r_{\nu} e^{i \theta_{\nu}}\right\}$ and $g(z)$ be the canonical product formed by zeros $\left\{\gamma_{\nu} e^{\imath\left(\theta_{\nu}+\alpha\right)}\right\}$, where $\alpha$ is a constant satisfying $0<\alpha \leqq \pi$. Let $F(z)$ be $f(z) / g(z)$. Then

$$
\begin{aligned}
\left|c_{m}(r)\right| & =-\frac{1}{2 m}\left|\sum_{r_{\nu} \leqq r}\left\{\left(\frac{r}{r_{\nu}}\right)^{m}-\left(\frac{r_{\nu}}{r}\right)^{m}\right\}\left(1-e^{-\imath m \alpha}\right) e^{-\imath m \theta_{\nu}}\right| \\
& \leqq \frac{\sqrt{2(1-\cos m \alpha)}}{2 m} \sum_{r_{\nu} \leqq r}\left\{\left(\frac{r}{r_{\nu}}\right)^{m}-\left(\frac{r_{\nu}}{r}\right)^{m}\right\} .
\end{aligned}
$$

Again by making use of Pólya peaks of the first kind, order $\rho$, for $N(r)=N(r, 0, f)$, we have

$$
\varliminf_{r \rightarrow \infty} \frac{m_{2}(r, F)^{2}}{N(r)^{2}} \leqq 16 \sum_{m=1}^{\infty}(1-\cos m \alpha) \frac{\rho^{4}}{\left(m^{2}-\rho^{2}\right)^{2}} .
$$

This is best possible. Especially, if $\alpha=\pi$, then we have Theorem 4 . 
$\S \mathbf{1 0}$. Let $F(z)$ be $f(z) g(z)$, where $f$ and $g$ are defined in $\S 9$. Then

$$
\begin{aligned}
\left|c_{m}(r)\right| & =\frac{1}{2 m}\left|\sum_{r_{\nu} \leqq r}\left\{\left(\frac{r}{r_{\nu}}\right)^{m}-\left(\frac{r_{\nu}}{r}\right)^{m}\right\}\left(1+e^{-\imath m \alpha}\right) e^{-\imath m \theta_{\nu}}\right| \\
& \leqq \frac{1}{2 m}(2+2 \cos m \alpha)^{1 / 2} \sum_{r_{\nu} \leqq r}\left\{\left(\frac{r}{r_{\nu}}\right)^{m}-\left(\frac{r_{\nu}}{r}\right)^{m}\right\} .
\end{aligned}
$$

Hence by the same method as in $\S 9$

$$
\varliminf_{r \rightarrow \infty} \frac{m_{2}(r, F)^{2}}{N(r, 0, F)^{2}} \leqq 1+\sum_{m=1}^{\infty}(1+\cos m \alpha) \frac{\rho^{4}}{\left(m^{2}-\rho^{2}\right)^{2}} .
$$

Especially for $\alpha=\pi$

$$
\varliminf_{r \rightarrow \infty} \frac{m_{2}(r, F)^{2}}{N(r, 0, F)^{2}} \leqq \frac{1}{8} \frac{\pi \rho(\pi \rho+\sin \pi \rho)}{\sin ^{2} \frac{\pi}{2} \rho} .
$$

Of course this is best possible. The last part is due to the following identities:

$$
\begin{gathered}
1+2 \sum_{m=1}^{\infty} \frac{\rho^{4}}{\left(\rho^{2}-m^{2}\right)^{2}}=\frac{1}{2} \frac{\pi \rho(\pi \rho+\cos \pi \rho \sin \pi \rho)}{\sin ^{2} \pi \rho}, \\
2 \sum_{p=1}^{\infty} \frac{\rho^{4}}{\left(\rho^{2}-(2 p-1)^{2}\right)^{2}}=\frac{1}{4} \frac{\pi \rho(\pi \rho-\sin \pi \rho)}{2 \cos ^{2} \frac{\pi}{2} \rho} .
\end{gathered}
$$

$\S 11$. Let $f(z)$ be the canonical product formed by zeros $\left\{r_{\nu}\right\}$ and $g(z)$ the canonical product formed by zeros $\left\{s_{\nu} e^{\imath \alpha}\right\}$, where $\alpha$ is a constant satisfying $0 \leqq \alpha \leqq \pi$. Let $F(z)$ be $f(z) g(z)$. Let $f_{1}(z)$ be the canonical product formed by zeros $\left\{r_{\nu}, s_{\nu}\right\}$ and $f_{1 \alpha}(z)$ be the canonical product formed by zeros $\left\{r_{\nu} e^{\imath \alpha}, s_{\nu} e^{\imath \alpha}\right\}$. Let $F_{1}(z)$ be $f_{1}(z) f_{1 \alpha}(z)$. Then

$$
\left|c_{m}(r ; F)\right|=\left|A_{m}+B_{m} e^{-\imath m \alpha}\right| .
$$

It is very easy to prove that

This gives

$$
2\left|A_{m}+B_{m} e^{-\imath m \alpha}\right| \geqq\left|A_{m}+B_{m}+\left(A_{m}+B_{m}\right) e^{-\imath m \alpha}\right| .
$$

$$
4 m_{2}(r, F)^{2} \geqq m_{2}\left(r, F_{1}\right)^{2} .
$$

By making use of Pólya peaks of the second kind, order $\rho$, for $N\left(r, 0, F_{1}\right)$ $=2 N(r, 0, F)$, we have

$$
\varliminf_{r \rightarrow \infty} \frac{N(r, 0, F)^{2}}{m_{2}(r, F)^{2}} \leqq\left(1+\sum_{m=1}^{\infty}(1+\cos m \alpha) \frac{\rho^{4}}{\left(m^{2}-\rho^{2}\right)^{2}}\right)^{-1 / 2}
$$

Especially for $\alpha=\pi$

$$
\varliminf_{r \rightarrow \infty} \frac{N(r, 0, F)}{m_{2}(r, F)} \leqq \frac{2 \sqrt{ } 2\left|\sin \frac{\pi}{2} \rho\right|}{\sqrt{\pi \rho(\pi \rho+\sin \pi \rho)}} .
$$


Again this is best possible.

\section{REFERENCES}

[1] EdRei, A. AND W.H.J. Fuchs, On the growth of meromorphic functions with several deficient values, Trans. Amer. Math. Soc. 93 (1959) 292-328.

[2] Kobayashi. T., On the deficiency of an entire function of finite genus, Kōdai Math. Sem. Rep. 27 (1976), 320-328.

[3] Kobayashi, T., On the lower order of an entire function, Kōdai Math. Sem. Rep. 27 (1976), 484-495.

[4] Miles, J. ANd D.F. Shea, An extremal problem in value-distribution theory, Quart. J. Math. Oxford 24 (1973), 377-383.

[5] Miles, J. AND D.F. SheA, On the growth of meromorphic functions having at least one deficient value, Duke Math. J. 43 (1976), 171-186.

[6] SHEA, D.F., On the Valiron deficiencies of meromorphic functions of finite order, Trans. Amer. Math. Soc. 124 (1966), 201-227.

Department of Mathematics

Tokyo Institute of Technology

OH-okayama, Meguro-ku, Tokyo, Japan 